\title{
Surgical Treatment of Rhinogenic Contact Point Headache: An Experience from a Tertiary Care Hospital
}

\author{
Aleksandar Peric ${ }^{1}$ Dejan Rasic ${ }^{1} \quad$ Ugljesa Grgurevic ${ }^{1}$ \\ ${ }^{1}$ Department of Otorhinolaryngology, Military Medical Academy, \\ Belgrade, Serbia \\ Address for correspondence Aleksandar Peric, MD, PhD, Department \\ of Otorhinolaryngology, Military Medical Academy, Crnotravska 17, \\ Belgrade 11000, Serbia (e-mail: alexneta@orion.rs). \\ Int Arch Otorhinolaryngol 2016;20:166-171.
}

\begin{abstract}
Introduction Even in the absence of inflammatory disease, facial pain often results from pressure of two opposing nasal mucosa surfaces.

Objectives The objective of this study is to assess the efficacy of surgical treatment of contact point headache.

Methods Our study enrolled patients with unilateral facial pain and without nasal/ paranasal sinus disease. We confirmed the presence of mucosal contact by nasal endoscopy and by computed tomography. Forty-two subjects with the three most common anatomical variations underwent complete evaluation: 17 with concha bullosa (CB), 11 with septal deviation (SD), and 14 with septal spur (SS). All participants were treated by topical corticosteroid, adrenomimetic, and antihistamine. The patients without improvement were treated surgically. We assessed the severity of pain using a Visual Analogue Score (VAS) before surgical treatment and one, six, twelve, and twenty-four months after.

Results The patients with SS had more severe facial pain in comparison with patients with CB $(p=0.049)$ and SD $(p=0.000)$. The subjects with CB had higher degree of facial pain than the ones with $S D(p=0.001)$. After an unsuccessful medical treatment

Keywords

- headache

- turbinates

- nasal septum

- otorhinolaryngologic surgical procedures and surgical removal of mucosal contacts, the decrease of headache severity was more intense in patients with CB and SS $(p=0.000)$ than in the patients with SD $(p=0.01)$. Conclusion Our results suggest that topical medications have no effects and that surgical removal of mucosal contacts could be effective in the treatment of contact point headache. The results of surgical treatment were better in cases of facial pain caused by SS and CB, than in those caused by SD.
\end{abstract}

\section{Introduction}

"Sinus headache", despite being a term commonly used by patients and primary care physicians, is often a misdiagnosis, which can lead to improper treatment. If a practitioner truly thinks the symptoms are due to abnormalities in the sinonasal region, a more accurate description would be "rhinogenic headache". ${ }^{1}$ Frontal headaches and facial pain can often arise as a result of acute or chronic inflammation of the lining of the paranasal sinuses and are usually associated with other sinonasal symptoms, such as nasal obstruction, muco-purulent secretions from the nose, postnasal discharge, and impaired sense of smell. ${ }^{2}$ Acute rhinosinusitis usually is characterized by purulent discharge in the nasal passages and a pain profile determined by the site of infection. ${ }^{3}$ Often a localized headache or toothache is present in acute sinusitis, and it is a symptom that distinguishes a sinus-related headache from other types of headaches, such as tension and migraine headaches. received

August 21, 2015

accepted

December 6, 2015

published online

February 17, 2016
DOI http://dx.doi.org/

10.1055/s-0036-1578808. ISSN 1809-9777.
Copyright $\odot 2016$ by Thieme Publicações License terms

Ltda, Rio de Janeiro, Brazil
(ब)(1) $\Theta$ 
Another way to distinguish between toothache and sinusitis pain derives from the fact that the pain in sinusitis usually is worsened by tilting the head forwards and by performing Valsalva maneuvers. ${ }^{3}$ However, sinusitis is over diagnosed as a cause of headache because of the belief that pain over the sinuses must be related to the sinuses. It is important to distinguish sinus pain from severe headaches that respond poorly to drug therapy, usually localized in the frontal and periorbital area or in the area of the face. However, medical history, rhinoscopic, and endoscopic examination, and radiologic findings do not indicate the presence of diseases of the mucous membranes in the nose and paranasal sinuses. ${ }^{4}$ It is usually a dull ache, accompanied by a sense of pressure, which oscillates in intensity and localization, or "pulsates" according to the nasal cycle. ${ }^{4}$

Stammberger and Wolf ${ }^{5}$ divided patients with headaches into three groups: (1) those with pain as a consequence of acute and chronic inflammation of the lining of the sinuses or barotrauma; (2) those which headaches are not caused by diseases of the sinuses, such as patients with allergic and nonallergic rhinitis, neuralgia, migraine or vascular problems; (3) those with pain of sinonasal origin, although its inflammatory origin cannot be confirmed. It is assumed that headache from the third group formed as a result of contacts and pressures between adjacent mucosal surfaces in the nasal cavity due to the presence of anatomical variations. The presence of such "contact points" between the deformity of the nasal septum and / or variation of the middle turbinate, among which is the most common pneumatization of the middle turbinate (concha bullosa) and the lateral wall of the nasal cavity, is considered to be a trigger of such atypical pain. ${ }^{6}$

On the other hand, despite intensive contacts of mucosal surfaces, the presence of facial pain is rare in chronic polypous rhinosinusitis due to the lack of innervation of nasal polyp mucous membrane. ${ }^{7,8}$

The aim of this retrospective study was to assess the role of surgical corrections of anatomical variations in the nasal cavity in the treatment of rhinogenic headaches that are not associated with inflammatory diseases of the sinonasal region.

\section{Patients and Methods}

The Ethics Committee of the Military Medical Academy approved the study. We provided detailed information about the study to the participants and obtained written informed consent from each one. We conducted a retrospective analysis of prospectively collected data on 238 patients presenting with chronic headache in our hospital. We recruited fifty-six
( $n=56$ ) patients with rhinogenic headache without symptoms and signs of acute and chronic sinonasal inflammation that underwent treatment at the Department of Otorhinolaryngology in the period from January 2007 to December 2014. We completely evaluated the effects of surgical treatment for contact point headache in forty-two $(n=42)$ patients, including the follow-up period of 24 months. Fourteen patients have not yet completed two years of surgery. All patients were examined and treated by one rhinologist. We included in the study only patients with facial pain, accompanied by a sense of pressure and present at least one year prior to start of the study. All patients had previously been on medical treatment for at least six months and only entered in the study if they were resistant to medical treatment. During the medical treatment, all 42 patients received three courses of 1.5 months therapy:

(1) fluticasone propionate aqueous nasal spray only (50 $\mu \mathrm{g}$, two actuations in each nostril, every morning);

(2) fluticasone propionate aqueous nasal spray (50 $\mu$ g, two actuations in each nostril, every morning) with oxymetazoline nasal spray ( $0.05 \%$, two puffs in each nostril, every evening);

(3) fluticasone propionate aqueous nasal spray ( $50 \mu \mathrm{g}$, two actuations in each nostril, every morning) with azelastine hydrochloride nasal spray $(137 \mu \mathrm{g}$, one puff in each nostril, every evening).

To decrease the adverse effects of the medications, the treatments were discontinued for a period of two weeks, just before the second and third course of the treatment. After the end of the third course of medical treatment, we found no adverse effects in our participants. However, the results of medical treatment regarding the removal or decrease of headache were not satisfactory. The results of medical treatment are presented in the - Table $\mathbf{1}$.

Only patients with normal neurological, ophthalmological, dental, and internal findings were included in this investigation. The presence of mucosal contacts was verified according to the findings of computed tomography (CT) in the corronal and axial sections (-Figs. 1 and 2) and endoscopy of the nasal cavity.

All patients had positive lidocaine tests. This means that, after the application of a piece of cotton wool soaked in the $5 \%$ solution of lidocaine for 15 minutes, there was a reduction in pain intensity of more than $50 \%$. We only included in the study patients with the three most commonly seen anatomical variations in the nasal cavities and, among 42 completely assessed patients, 17 presented concha bullosa of the middle turbinate, 11 had deviation of the nasal septum, and 14 had

Table 1 Results of medical treatment

\begin{tabular}{|l|l|l|l|l|}
\hline Anatomical variation & Before treatment & 2 months after & 6 months after & $\boldsymbol{p}$ \\
\hline Concha bullosa & $7.65 \pm 1.23$ & $7.51 \pm 0.92$ & $7.18 \pm 0.73$ & $>0.05$ \\
\hline Septal deviation & $6.25 \pm 0.98$ & $6.09 \pm 1.27$ & $5.91 \pm 0.94$ & $>0.05$ \\
\hline Septal spur & $8.37 \pm 1.18$ & $8.20 \pm 0.86$ & $7.93 \pm 0.92$ & $>0.05$ \\
\hline Total & $7.41 \pm 1.27$ & $7.26 \pm 0.96$ & $7.10 \pm 1.14$ & $>0.05$ \\
\hline
\end{tabular}




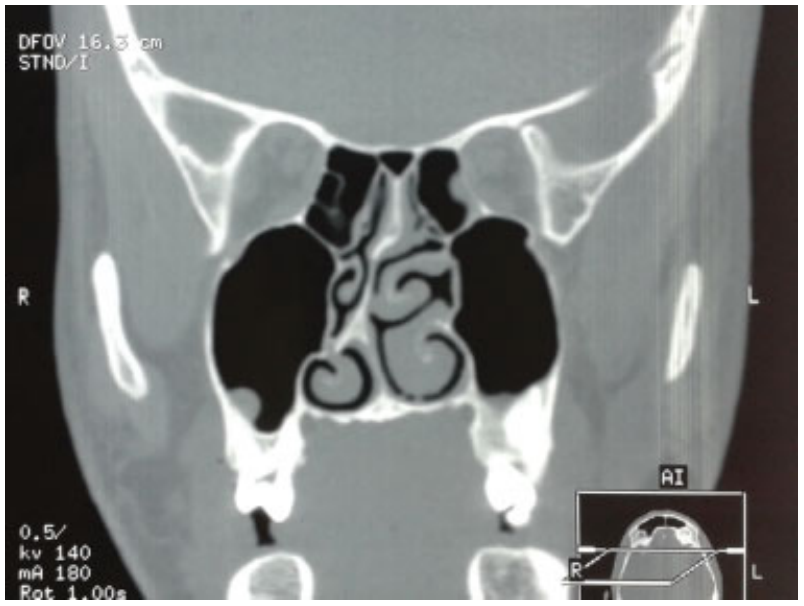

Fig. 1 Corronal CT scan showing intensive mucosal contact between the peak of septal spur and the upper surface of the right inferior turbinate.

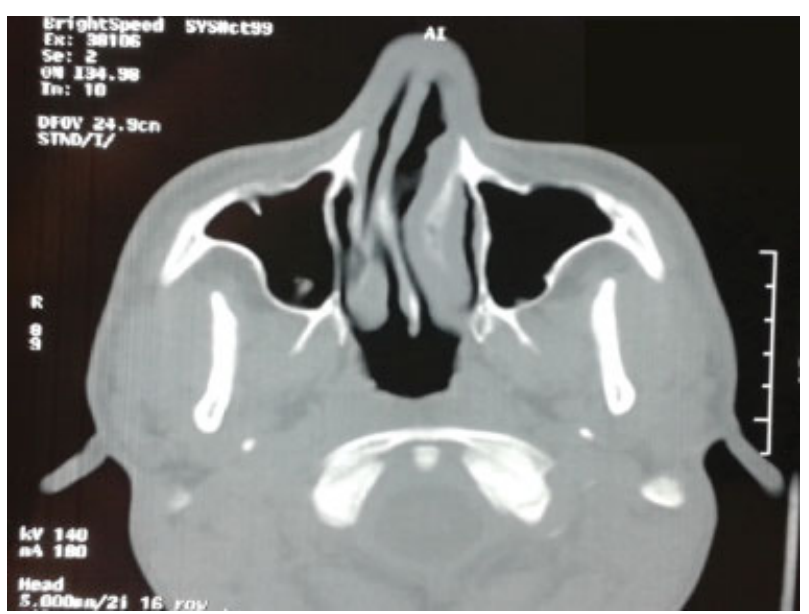

Fig. 2 Axial CT scan showing mucosal contact between the peak of septal spur and the right inferior nasal turbinate mucosa.

septal spur. Subjects with more than one mucosal contact (for example, because of septal deviation and concha bullosa at the same time) were excluded from the study. Moreover, we medically and surgically treated several patients with contact point headache associated with other anatomical variations in the same period: 3 with paradoxically curved middle turbinate, 3 with concha bullosa of the superior turbinate, 2 with enlarged ethmoid bulla, and one with bifurcate middle turbinate. In all patients, the mucosal contacts between middle turbinate/superior turbinate and nasal septum, and bulla ethmoidalis and middle turbinate were demonstrated by endoscopic examination and CT scan. After an unsuccessful medical treatment, the patients with paradoxically curved middle turbinate and bifurcate middle turbinate were treated by endoscopic medialization/lateralization of the middle turbinate, whereas the patients with concha bullosa of the superior turbinate were treated by lateral resection of the pneumatized concha. Patients with enlarged ethmoid bulla underwent bullectomy. However, due to the low frequency of above mentioned anatomical configurations, we could not include them in the complete evaluation and statistical analysis.

The mucosal contacts in 42 completely evaluated subjects were removed under general anesthesia using an endoscopic approach (lateral concha bullosa resection, endoscopic septoplasty) or conventional endonasal procedures (septoplasty according to Cottle). We evaluated the intensity of pain by Visual Analogue Score (VAS) before medical treatment, and at two months and six months after therapy by medications, as well as before surgical treatment, one month, six months, one year and two years after the surgery. The facial pain was scored as follows: 0 , absence of pain; $1-3$, mild pain; 4-6, moderately severe pain; $7-9$, severe pain; 10 , the worse pain imaginable. The follow-up period for complete evaluation was 24 months after surgical treatment. We assessed two additional parameters: duration of headaches (number of hours with pain for 24 hours) and the frequency of headaches (number of days with pain in one month).

Criteria for exclusion from the study were: allergic and non-allergic rhinitis, acute and chronic rhinosinusitis, mucoceles and pyoceles of the paranasal sinuses, sinonasal tumors, systemic or rheumatic diseases, previous surgical procedures in the nose or sinuses.

\section{Statistical Analysis}

We analyzed data using SPSS 17.0 for Windows. We used oneway ANOVA (analysis of variance) test with Dunn-Bonferroni correction for comparison between the groups. We used the Paired $t$ test (two-tailed $t$ test) for the evaluation of variable means in each group. Results are presented as mean \pm standard deviation (SD) and we assumed a significance level of 0.05 .

\section{Results}

Among forty-two $(n=42)$ completely evaluated patients, 22 of them (52.38\%) were male, and 20 (47.61\%) were female. The youngest participant was 18 and the oldest 65 years old and the mean age of patients was $37.48 \pm 12.17$ years. Data on the results of medical treatment are presented in - Table 1 .

When we compared the intensity of headache between the three groups, we found that it was higher in patients with septal spur in relation to patients with nasal septum deviation $(p=0.000)$ and patients with concha bullosa $(p=0.049)$. Also, in patients with concha bullosa, pain was more intensive as compared with those with the nasal septal deviation $(p=0.001)$. The results regarding the intensity of headaches can be seen in - Table 2 .

Comparing the duration of headache (number of hours with headache during 24 hours), we found that it is longer in patients with septal spur compared with patients with septal deviation $(p=0.000)$ and in patients with concha bullosa compared with patients with septum deviation $(p=0.000)$. There was no statistically significant difference when we compared the patients with septal spur and those with concha bullosa ( $p=0.099$ ). Data relating to the duration of pain are presented in -Table 2. 
Table 2 Clinical parameters before surgical treatment

\begin{tabular}{|c|c|c|c|}
\hline $\begin{array}{l}\text { Anatomical } \\
\text { variation }\end{array}$ & Mean \pm SD & Minimum & Maximum \\
\hline \multicolumn{4}{|c|}{ Intensity of headache } \\
\hline Concha bullosa & $7.18 \pm 0.73$ & 6 & 8 \\
\hline Septal deviation & $5.91 \pm 0.94$ & 5 & 8 \\
\hline Septal spur & $7.93 \pm 0.92$ & 6 & 9 \\
\hline Total & $7.10 \pm 1.14$ & 5 & 9 \\
\hline \multicolumn{4}{|c|}{ Duration of headache } \\
\hline Concha bullosa & $10.35 \pm 2.32$ & 5 & 13 \\
\hline Septal deviation & $6.09 \pm 1.30$ & 4 & 8 \\
\hline Septal spur & $11.93 \pm 2.20$ & 8 & 15 \\
\hline Total & $9.76 \pm 3.07$ & 4 & 15 \\
\hline \multicolumn{4}{|c|}{ Frequency of headache } \\
\hline Concha bullosa & $10.35 \pm 2.32$ & 5 & 13 \\
\hline Septal deviation & $6.09 \pm 1.30$ & 4 & 8 \\
\hline Septal spur & $11.93 \pm 2.20$ & 8 & 15 \\
\hline Total & $9.76 \pm 3.07$ & 4 & 15 \\
\hline
\end{tabular}

Finally, when we compared the frequency of pain (the number of days with headache in a month), we observed that it is higher in the group of patients with septal spur in comparison to those with septal deviation $(p=0.000)$, as well as in the group with concha bullosa compared the group with septal deviation $(p=0.001)$. On the other hand, no difference was found in the frequency of headache between the patients with concha bullosa and septal spur $(p=0.068)$. Data on the frequency of pain are presented in - Table 2 .

In patients with concha bullosa, the removal of mucosal contacts was performed by endoscopic resection of the lateral portion of the concha bullosa (endoscopic lateral laminectomy) and, in patients with septal deviation, by conventional endonasal procedure (septoplasty according to Cottle). In patients with septal spur of small area $(n=8)$, the procedure is done under the endoscopic guidance (endoscopic septoplasty), and in those with broad-based area $(n=6)$, we performed the conventional septoplasty by Cottle. Although the results of surgical treatment were very good, we could not register any patient who has come with a complete withdrawal of headache. In five $(n=5)$ patients (11.90\%), the facial pain did not improve. Of these patients, three had septal deviation and two concha bullosa before surgery. After the surgical removal of mucosal contacts, headache intensity reduction was more pronounced in the patients with concha bullosa $(p=0.000)$ and septal spur $(p=0.000)$ than in the patients with septal deviation $(p=0.01)$. The same trend of decreasing pain intensity was presented at 6 months, 12 months, and 24 months after the surgical treatment. Data on the results of surgical treatment are presented in $\boldsymbol{- T a b l e} \mathbf{3}$.

\section{Discussion}

Rhinogenic contact point headache pathogenesis is still the subject of controversy. Regarding the fact that the endonasal contact points may be present in people who do not have a headache, the causal link between these contacts and headaches is not easily established. The debate over the contact point headaches within the scientific community has a long history. Back in 1948, Wolff hypothesized that pain can be caused by contacts between the middle turbinate and other regions of the nasal cavity. ${ }^{9}$ Wolff's results showed that stimulation of various points of the nasal mucosa can cause pain that was felt in the cutaneous distribution of ophthalmic (V1) and maxillary (V2) division of the trigeminal nerve. ${ }^{9}$ This is particularly present during the stimulation of the anterior part of the middle turbinate, especially in cases of extensive pneumatization (extensive type of concha bullosa). ${ }^{10,11}$

The frontal portion of the middle turbinate is innervated by anterior ethmoid nerve, which is a branch of ophtalmic nerve. Stimulation of this anterior segment leads to pain sensation in the skin distribution of infratrochlear and supratrochlear nerve, which are also branches of the ophthalmic nerve. The mucosa of the nasal septum have identical innervation and its stimulation obtained pain with similar localization. So far, studies have explained that neuropeptides substance P and CGRP (Calcitonin Gene Related Peptide) are mediators of rhinogenic pain. ${ }^{5}$ Substance $P$ was isolated in 1931, but, in 1971, researchers found it to be an undecapeptide (polypeptide chain consisting of 11 amino acids). The hypothesis of polymodal receptors suggests that different caloric, chemical, and mechanical irritantś (such as pressure on the mucosa) stimulation of the nasal mucosa may cause the release of neuropeptides through the central orthodromic impulse and peripheral local, antidromic impulse. Locally, neuropeptides lead to vasodilatation and edema of mucosal membrane, which further intensifies the pressure of contact surfaces. The release of neuropeptides in the central nervous system causes the sensation of pain, which is similar to migraine without aura. The onset and duration of pain coincide with the beginning and duration of the nasal cycle. ${ }^{5}$

It appears that the contact between the mucosal surfaces causes pain stronger than one that occurs as a result of infection and inflammation. Stammberger and Wolf ${ }^{5}$

Table 3 Results of surgical treatment

\begin{tabular}{|l|l|l|l|l|l|l|}
\hline Anatomical variation & Before surgery & $\mathbf{1}$ month after & $\mathbf{6}$ months after & 12 months after & 24 months after & $\boldsymbol{p}$ \\
\hline Concha bullosa & $7.18 \pm 0.73$ & $2.95 \pm 1.02$ & $2.52 \pm 1.25$ & $2.38 \pm 0.94$ & $2.34 \pm 0.88$ & 0.000 \\
\hline Septal deviation & $5.91 \pm 0.94$ & $3.05 \pm 1.43$ & $3.01 \pm 0.95$ & $2.85 \pm 1.28$ & $2.81 \pm 1.34$ & 0.01 \\
\hline Septal spur & $7.93 \pm 0.92$ & $2.43 \pm 0.97$ & $2.28 \pm 1.05$ & $1.98 \pm 0.75$ & $1.82 \pm 0.69$ & 0.000 \\
\hline Total & $7.10 \pm 1.14$ & $3.12 \pm 1.04$ & $2.71 \pm 1.02$ & $2.43 \pm 0.88$ & $2.38 \pm 0.78$ & 0.001 \\
\hline
\end{tabular}


measured the concentration of substance $\mathrm{P}$ in the nasal mucosa and concluded that it is higher in healthy mucosa compared with chronically inflamed hyperplastic mucosa and mucosa of the nasal polyps. The explanation for this is that the respiratory epithelium of the nasal mucosa produces enzyme neural endopeptidase (NEP), which is able to degrade substance P. However, this degrading action of NEP may be disturbed by limited areas of mucosal contact where the epithelium is affected and the basement membrane (which contains a larger number of afferent nerves) is exposed. ${ }^{12}$ This leads to an increase in the intensity of pain. ${ }^{12}$

In our study, we found the most intensive pain sensation in patients with septal spur. Duration and frequency of headaches are highest in patients with mucosal contacts associated with septal spur. It seems that pressure between mucosal surfaces in small area of septal spur peak results in more intensive sense of pain than in the cases of septal deviation and concha bullosa. We suggest that this form of mucosal contact may imply more intense production and local mucosal liberation of substance $\mathrm{P}$ and other neuropeptides.

The diagnosis of contact point headache requires a multidisciplinary approach. Patients with headaches without findings of inflammation of mucosal membranes of the sinonasal region should be examined by a neurologist, ophthalmologist, dentist, and internist to exclude other causes. After rhinoscopic and endoscopic examination and $\mathrm{CT}$ of the paranasal sinuses, it is very important to perform the lidocaine test. It can help not only the diagnosis of this type of headache, but also acts as an indicator of the success of surgical removal of mucosal contact. ${ }^{13} \mathrm{CT}$ is able to recognize some pathologic findings that cannot be found on physical examination and is helpful to decide the location and the type of the surgery. ${ }^{14}$ Endoscopic surgical treatment affords superior visualization of mucosal contacts, which is important for their limited resection and allows for a more controlled and precise surgery, with minimal trauma.

There are several studies that have analyzed the success of the surgery of contact point headache. The criteria for inclusion and the results were different from study to study. The biggest series, which was presented by Huang et $\mathrm{al}^{15}$ included 66 patients divided into three groups: with deviation of the nasal septum, with concha bullosa, and with orbitoethmoidal (Haller's) cell. After the surgical treatment, the authors found a reduction of intensity and frequency of headache in $81.8 \%$ of the patients. Parsons and Batra ${ }^{16}$ demonstrated an improvement rate of $91 \%$ in a retrospective study including 34 subjects with contact between the septum and nasal turbinates. Sadeghi et al published similar results (improvement in $93.3 \%$ of patients) with similar groups for a total of 30 patients. ${ }^{17}$ Our results, with an improvement rate of $88.10 \%$, is generally in accordance with previously published results. In none of these studies do the patients require any medication after the surgical treatment. We found one study with different results published by WelgeLuessen et al. ${ }^{18}$ This investigation included a total of 20 patients with headaches that were caused by contacts between the middle turbinate and the nasal septum or between the middle turbinate and ethmoid bulla. The follow-up was 10 years. After such a long follow-up, the rate of improvement was "only"
$65 \%{ }^{18}$ This points to the conclusion that the criteria for selecting these patients should be better standardized and that the period of patients' monitoring after surgical treatment should be considerably longer.

Yamohammadi et $\mathrm{al}^{19}$ compared the effects of medical and surgical treatment of contact point headaches associated by concha bullosa. They found that simple surgical resection of lateral portion of concha bullosa to eliminate the contact points between the nasal mucosal surfaces is superior and more cost efficient in comparison to three courses of 1.5 months medical management with fluticasone propionate intranasal spray and oral pseudoephedrin. ${ }^{19}$ The results of our study showed no improvement in contact point headache intensity after the three courses of medical treatment. A previous study presented by Baroody et $\mathrm{al}^{20}$ clearly demonstrated that the addition of oxymetazoline nasal spray adds to the effectiveness of intranasal corticosteroid administration in patients with perennial allergic rhinitis. In a group of patients with combined treatment, the authors found higher reduction in nasal congestion symptom score in comparison to the group receiving only corticosteroid therapy. They explained such effect by the combined antiedematous action of both medications. However, such combined local therapy has no effect in the reduction of contact point headache. Therefore, the combined fluticasone propionate - azelastine hydrochloride treatment also showed no improvement in headache intensity. In clinical studies, azelastine decreased substance P levels in allergic and non-allergic rhinitis patients over a 4week treatment period by inhibition of substance $P$ release and activity. ${ }^{21}$ As substance $P$ represents one of the most important pain neurotransmitters, we believed that use of a substance $P$ inhibitor can decrease the pain severity in our patients.

According to some authors, the phenomenon of contact point headache is controversial. Abu-Bakra and Jones ${ }^{22}$ stimulated nasal structures with mechanical probe and substance $\mathrm{P}$ and caused local pain, but could not elicit referred facial pain. They found similar incidence of contact points (4\%) in those with and without facial pain. In patients with unilateral facial pain, the contact point was seen on the contralateral side in $50 \%$ of patients. ${ }^{20}$ Harrison and Jones ${ }^{23}$ performed a systematic review of the literature with 973 consecutive patients. They found that the majority of people with contact points experience no facial pain and concluded that the presence of contact point is not a good predictor of facial pain. They showed that the removal of a contact point rarely results in the total elimination of headache, which is in accordance with our results. Finally, according to Harrison and Jones, improvement in postoperative symptoms following the removal of contact points in some patients may be explained by cognitive dissonance or neuroplasticity. ${ }^{23}$

\section{Conclusion}

Our results demonstrated much better results from surgical therapy for contact point headache in comparison with local medical treatment modalities. Surgical removal of contact points in the nasal cavity can be effective in treating the headache. Contact point headache has the highest severity, duration, and frequency in patients with mucosal contacts 
associated with septal spur. The results of surgical treatment are better in cases of headache associated with the presence of septal spur and concha bullosa than in those associated with nasal septal deviation.

\section{References}

1 Patel ZM, Kennedy DW, Setzen M, Poetker DM, DelGaudio JM. "Sinus headache": rhinogenic headache or migraine? An evidencebased guide to diagnosis and treatment. Int Forum Allergy Rhinol 2013;3(3):221-230

2 Patel ZM, Setzen M, Poetker DM, DelGaudio JM. Evaluation and management of "sinus headache" in the otolaryngology practice. Otolaryngol Clin North Am 2014;47(2):269-287

3 Marmura MJ, Silberstein SD. Headaches caused by nasal and paranasal sinus disease. Neurol Clin 2014;32(2):507-523

4 Roozbahany NA, Nasri S. Nasal and paranasal sinus anatomical variations in patients with rhinogenic contact point headache. Auris Nasus Larynx 2013;40(2):177-183

5 Stammberger $\mathrm{H}$, Wolf $\mathrm{G}$. Headaches and sinus disease: the endoscopic approach. Ann Otol Rhinol Laryngol Suppl 1988; 134:3-23

6 Herzallah IR, Hamed MA, Salem SM, Suurna MV. Mucosal contact points and paranasal sinus pneumatization: Does radiology predict headache causality? Laryngoscope 2015;125(9): 2021-2026

7 Eweiss AZ, Lund VJ, Barlow J, Rose G. Do patients with chronic rhinosinusitis with nasal polyps suffer with facial pain? Rhinology 2013;51(3):231-235

8 Andrade GC, Fujise LH, Fernandes AM, Azoubel R. Rhinosinusal polyposis and inverted papilloma: a morphometric comparative study. Int Arch Otorhinolaryngol 2015;19(3):196-199

9 Wolff HG. The nasal, paranasal, and aural structures as sources of headache and other pain. In: Wolff HG editor. Headache and other head pain. New York: Oxford University Press; 1948. p. 532-560.

10 Perić A, Sotirović J, Baletić N, Kozomara R, Bijelić D, Rasić D. [Concha bullosa and the nasal middle meatus obstructive syndrome]. Vojnosanit Pregl 2008;65(3):255-258
11 Peric A, Baletic N, Sotirovic J. A case of an uncommon anatomic variation of the middle turbinate associated with headache. Acta Otorhinolaryngol Ital 2010;30(3):156-159

12 Clerico DM, Evan K, Montgomery L, Lanza DC, Grabo D. Endoscopic sinonasal surgery in the management of primary headaches. Rhinology 1997;35(3):98-102

13 Mokbel KM, Abd Elfattah AM, Kamal S. Nasal mucosal contact points with facial pain and/or headache: lidocaine can predict the result of localized endoscopic resection. Eur Arch Otorhinolaryngol 2010;267(10):1569-1572

14 Karataş D, Yüksel F, Sentürk M, Doğan M. The contribution of computed tomography to nasal septoplasty. J Craniofac Surg 2013; 24(5):1549-1551

15 Huang HH, Lee TJ, Huang CC, Chang PH, Huang SF. Non-sinusitisrelated rhinogenous headache: a ten-year experience. Am J Otolaryngol 2008;29(5):326-332

16 Parsons DS, Batra PS. Functional endoscopic sinus surgical outcomes for contact point headaches. Laryngoscope 1998;108(5):696-702

17 Sadeghi M, Saedi B, Ghaderi Y. Endoscopic management of contact point headache in patients resistant to medical treatment. Indian J Otolaryngol Head Neck Surg 2013;65(Suppl 2):415-420

18 Welge-Luessen A, Hauser R, Schmid N, Kappos L, Probst R. Endonasal surgery for contact point headaches: a 10-year longitudinal study. Laryngoscope 2003;113(12):2151-2156

19 Yarmohammadi ME, Ghasemi H, Pourfarzam S, Nadoushan MR, Majd SA. Effect of turbinoplasty in concha bullosa induced rhinogenic headache, a randomized clinical trial. J Res Med Sci 2012; 17(3):229-234

20 Baroody FM, Brown D, Gavanescu L, DeTineo M, Naclerio RM. Oxymetazoline adds to the effectiveness of fluticasone furoate in the treatment of perennial allergic rhinitis. J Allergy Clin Immunol 2011;127(4):927-934

21 Lieberman PL, Settipane RA. Azelastine nasal spray: a review of pharmacology and clinical efficacy in allergic and nonallergic rhinitis. Allergy Asthma Proc 2003;24(2):95-105

22 Abu-Bakra M, Jones NS. Prevalence of nasal mucosal contact points in patients with facial pain compared with patients without facial pain. J Laryngol Otol 2001;115(8):629-632

23 Harrison L, Jones NS. Intranasal contact points as a cause of facial pain or headache: a systematic review. Clin Otolaryngol 2013; $38(1): 8-22$ 